\title{
IAMJ
}

INTERNATIONAL

AYURVEDIC

MEDICAL JOURNAL

Review Article

ISSN: 2320-5091

Impact Factor: 6.719

\section{A SYSTEMATIC REVIEW ON SELECTED MEDICINAL PLANTS USED IN THE MANAGEMENT OF NEERCHURUKKU (UTI)}

\author{
$\underline{\text { Kavery Piratheepkumar }}^{*}$, A. Manoharan ${ }^{2}$, A. Rajarajeshwari ${ }^{3}$
}

${ }^{1}$ PG Scholar, Department of General Medicine, Govt. Siddha Medical College, Tirunelveli, Tamil Nadu, India

${ }^{2}$ Professor \& HOD, Department of General Medicine, Govt. Siddha Medical College, Tirunelveli, Tamil Nadu, India

${ }^{3}$ Lecturer, Research Methodology and Bio-medical Statistics, Govt. Siddha Medical College, Tirunelveli, Tamil Nadu, India

Corresponding Author: bkavery405@gmail.com

\section{https://doi.org/10.46607/iamj0209032021}

(Published online: March 2021)

Open Access

(C) International Ayurvedic Medical Journal, India 2021

Article Received: 28/01/2021 - Peer Reviewed: 08/02/2021 - Accepted for Publication: 28/02/2021

Check for updates

\section{ABSTRACT}

The Neerchurukku (UTI) can be corrected with urinary tract infection (UTI) in modern medicine according their sign and symptoms. As per WHO an estimated 50\% of females were reporting UTI. It's affecting 150 million people in year in around the world. Aim of this study is to collect and review the medicinal plants used in the management of Neerchurukku noi. The Objectives were to list out the medicinal plants used for management of Neerchurukku noi, and to document the pharmacological studies of plants which are used for management of Neerchurukku noi. Research Type: Review study by systematic review method. study performed in Library, Government Siddha Medical College, Palayamkottai and PubMed, Google search from the published journal articles. This research finally concluded as collected and reviewed the 39 medicinal plants used in the management of Neerchurukku noi (UTI). listed out the 39 medicinal plants used for management of Neerchurukku no. Fabaceae family's plants were 5, Asteracea and Malvaceae family plants were 4, Lamiaceae family's plants were 3, Apiaceae, Euphorbiaceae and Mimosaceae family's plants were 2. Other 1 plant in various 17 families out of the 24 families. Plant's Parts used for management of UTS as; leaves were most used in 18 plants (38\%), roots were used in 9 plants (19\%), barks were 7 plants (15\%), 4 plants of fruits and seeds used (9\%) and aerial parts, whole plants, stem flower and gum 
were used by only one plant (2\%). Documented Antibacterial, Antifungal, Anti-plasmodial, Antimicrobial, Antioxidant, inhibits $\alpha$-glucosidase, Antiviral and Anti-inflammatory pharmacological actions of plants which are used for management of Neerchurukku noi. Decoction was most common prepared form of medicine to manage the Neerchurukku noi (UTI) by these medicinal plants.

Keywords: Neerchurukku noi, Urinary Tract Infection (UTI), Medicinal Plants

\section{INTRODUCTION}

A Siddha aspect, Yogi describe the disease Neer Noikal in his text yogi Vaithya Chinthamani is Neerinai perukkal noi and Neerinai Arukkal noi The Neerchurukku is described in the category of Neerinai Arukkal noi ${ }^{28}$. Neerchurukku can be corrected in modern aspect is urinary tract infection (UTI) according to their sign and symptoms. Urinary tract infection is one of the infectious diseases affecting both sexes, but most common in females. ${ }^{20}$ As per WHO an estimated $50 \%$ of females reporting having had a UTI some points of their lives ${ }^{17}$. UTI affecting 150 million people each year worldwide. UTI is very common disease in the society particularly in a summer season. ${ }^{4}$

\section{Causes of UTI:}

- Inadequate or consume small amount of oral fluids

- Retention of urine

- Renal stone

- Diabetic

- Un hygienic Sexual activities

- Prostatitis in Male 7,3,16,17,18,20

The severe UTI are occurred more frequently in diabetic patients. In a study from Europe, asymptomatic bacteria were more prevalent among women with diabetes $(26 \%)$ than in women without diabetes $(6 \%)^{1,2,5,7,9,10,24}$ Diabetic patients are at a high risk of development of UTIs. ${ }^{10,12,13}$ Antimicrobial therapies should be guided both by in vitro sensitivity and clinical response. In Siddha system of medicine prescribed the management to Neerchurukku from ancient era by the available natural resources such as plants, animal products and metals \& minerals. It has many evidence in manuscripts, textbooks and published articles now.

\section{Materials and Methods}

Research Type: Review study by systematic review method
Details search will be performed in Library, Government Siddha Medical College, Palayamkottai and Pub med, Google search from the published journal articles. Quality Assurance: Following procedures are conducted in time with the good planning by primary investigator. Whole research work had done with time frame schedule. The steps include: Protocol development $->$ Data collection $->$ Data analysis with RCS of references.

Ethical Issue: The study is to be carried out in research articles. It does not involve any physical examinations or instrumented. Therefore, this study does not need IEC approval. 


\section{Result}

Table 1: List of Medicinal plants used in UTI

\begin{tabular}{|c|c|c|c|c|c|c|c|}
\hline $\begin{array}{l}\text { Sl. } \\
\text { No. }\end{array}$ & Botanical name & $\begin{array}{l}\text { Plant } \\
\text { family }\end{array}$ & $\begin{array}{l}\text { Plant } \\
\text { part used }\end{array}$ & $\begin{array}{l}\text { Plant used } \\
\text { indica- } \\
\text { tions }\end{array}$ & application & $\begin{array}{l}\text { Chemical composi- } \\
\text { tion }\end{array}$ & References \\
\hline 1 & $\begin{array}{l}\text { Abelmoschus es- } \\
\text { culentus } \\
\text { Moench }\end{array}$ & $\begin{array}{l}\text { Malva- } \\
\text { ceae }\end{array}$ & Fruit & $\begin{array}{l}\text { Skin in- } \\
\text { fections, } \\
\text { UTIs }\end{array}$ & $\begin{array}{l}\text { With sugar } \\
\text { seedless fruits } \\
\text { taken orally }\end{array}$ & $\begin{array}{l}\text { Glycosides, Terpe- } \\
\text { noids, Tannins }\end{array}$ & $\begin{array}{l}\text { Afolayan AJ, et al. } \\
\text { (2014) }\end{array}$ \\
\hline 2 & $\begin{array}{l}\text { Abutilon indicum } \\
\text { L. }\end{array}$ & $\begin{array}{l}\text { Malva- } \\
\text { ceae }\end{array}$ & $\begin{array}{l}\text { Root, } \\
\text { leaves }\end{array}$ & UTIs & $\begin{array}{l}\text { Powder of } \\
\text { leaves and roots } \\
\text { taken orally }\end{array}$ & $\begin{array}{l}\text { Alkaloids, Steroids, } \\
\text { Flavonoids, Sterols, } \\
\text { Terpenoids, Phenols, } \\
\text { Glycosides, Saponins }\end{array}$ & Sharma A, et al. (2009) \\
\hline 3 & $\begin{array}{l}\text { Acacia gerrardii } \\
\text { Benth. }\end{array}$ & $\begin{array}{l}\text { Mimosa- } \\
\text { ceae }\end{array}$ & Bark & $\begin{array}{l}\text { Stomach } \\
\text { infections, } \\
\text { UTI }\end{array}$ & Decoction & $\begin{array}{l}\text { Infections of the up- } \\
\text { per respiratory system }\end{array}$ & Kokwaro (1976) \\
\hline 4 & $\begin{array}{l}\text { Acacia nilotica } \\
\text { (L.) Delile }\end{array}$ & $\begin{array}{l}\text { Mimosa- } \\
\text { ceae }\end{array}$ & $\begin{array}{l}\text { Bark, } \\
\text { Leaves, } \\
\text { Gum, }\end{array}$ & $\begin{array}{l}\text { Stomach } \\
\text { infections, } \\
\text { Malaria, } \\
\text { UTIs }\end{array}$ & $\begin{array}{l}\text { Decoction, } \\
\text { Gum Paste and } \\
\text { leaves with } \\
\text { cow's milk are } \\
\text { taken orally. } \\
\text { Powder of Bark }\end{array}$ & $\begin{array}{l}\text { Antibacterial, H. py- } \\
\text { lori urease inhibition, } \\
\text { Flavonoids, Cardiac } \\
\text { Glycosides, Anthra- } \\
\text { quinones, Tannins, } \\
\text { Saponins }\end{array}$ & $\begin{array}{l}\text { Amin et al.(2013); Ven- } \\
\text { kataswamy et al.(2010) }\end{array}$ \\
\hline 5 & $\begin{array}{l}\text { Ananus comosus } \\
\text { (L.) Merr. }\end{array}$ & $\begin{array}{l}\text { Brome- } \\
\text { liaceae }\end{array}$ & $\begin{array}{l}\text { Leaves, } \\
\text { fruit }\end{array}$ & $\begin{array}{l}\text { Skin in- } \\
\text { fections, } \\
\text { UTIs }\end{array}$ & $\begin{array}{l}\text { Leaves and fruit } \\
\text { juice in com- } \\
\text { bined form }\end{array}$ & $\begin{array}{l}\text { Alkaloids, Phenols, } \\
\text { Flavonoids, Glyco- } \\
\text { sides, Tannin, Phy- } \\
\text { tosterols, }\end{array}$ & Jaradat NA, et al., (2017) \\
\hline 6 & $\begin{array}{l}\text { Andrographis pa- } \\
\text { niculate Wall. ex. } \\
\text { Nees. }\end{array}$ & $\begin{array}{l}\text { Acan- } \\
\text { thaceae }\end{array}$ & Leaves & $\begin{array}{l}\text { Skin in- } \\
\text { fections, } \\
\text { UTIs }\end{array}$ & $\begin{array}{l}\text { Extract of Fresh } \\
\text { leaves }\end{array}$ & $\begin{array}{l}\text { Alkaloids, Anthra- } \\
\text { cene, Steroids, Glyco- } \\
\text { sides, Quinines, Fla- } \\
\text { vonoids, Phenols, } \\
\text { Tannins }\end{array}$ & Clark TE, (1997) \\
\hline 7 & $\begin{array}{l}\text { Apium graveo- } \\
\text { lens L. }\end{array}$ & Apiaceae & $\begin{array}{l}\text { Aerial } \\
\text { part }\end{array}$ & $\begin{array}{l}\text { Skin in- } \\
\text { fections, } \\
\text { UTIs }\end{array}$ & $\begin{array}{l}\text { Extract of fresh } \\
\text { leaves and fruits }\end{array}$ & $\begin{array}{l}\text { Alkaloids, Tannins, } \\
\text { Steroids, Flavonoids, } \\
\text { Terpenoids, Phenols, }\end{array}$ & Turner A, et al., (2007) \\
\hline 8 & $\begin{array}{l}\text { Asparagus afri- } \\
\text { canus Lam. }\end{array}$ & $\begin{array}{l}\text { Aspara- } \\
\text { gaceae }\end{array}$ & Root & UTIs & $\begin{array}{l}\text { Poultice, decoc- } \\
\text { tion }\end{array}$ & $\begin{array}{l}\text { Anti-plasmodial, sex- } \\
\text { ual diseases }\end{array}$ & $\begin{array}{lr}\text { Debella et al.(1999); } \\
\text { Oketch-Rabah } \\
\text { al.(1997);Neu- } \\
\text { winger(1998) }\end{array}$ \\
\hline 9 & $\begin{array}{l}\text { Azadirachta in- } \\
\text { dica A. Juss. }\end{array}$ & $\begin{array}{l}\text { Me- } \\
\text { liaceae }\end{array}$ & $\begin{array}{l}\text { Fruit, } \\
\text { leaves, } \\
\text { Bark }\end{array}$ & $\begin{array}{l}\text { Skin in- } \\
\text { fections, } \\
\text { UTIs }\end{array}$ & $\begin{array}{l}\text { Powder of bark } \\
\text { and leaves, } \\
\text { fresh fruits are } \\
\text { taken }\end{array}$ & $\begin{array}{l}\text { Alkaloids, Polyphe- } \\
\text { nols, Saponins, Fla- } \\
\text { vonoid, Anthraqui- } \\
\text { nones, Cardiac glyco- } \\
\text { sides, Terpenoids, } \\
\text { Terpenes, Steroids, } \\
\text { Tannins, }\end{array}$ & $\begin{array}{l}\text { Bussmann RW, et al., } \\
\text { (2006) }\end{array}$ \\
\hline 10 & Bidens pilosa $\mathrm{L}$. & $\begin{array}{l}\text { Aster- } \\
\text { aceae }\end{array}$ & $\begin{array}{l}\text { Whole } \\
\text { herb }\end{array}$ & $\begin{array}{l}\text { Skin in- } \\
\text { fections, } \\
\text { UTIs }\end{array}$ & $\begin{array}{l}\text { Extract of entire } \\
\text { plants is taken }\end{array}$ & $\begin{array}{l}\text { Alkaloids Flavonoids, } \\
\text { Steroids, Anthraqui- } \\
\text { nones, Tannins, Gly- } \\
\text { cosides, Saponins, }\end{array}$ & Nie Y, et al., (2013) \\
\hline
\end{tabular}




\begin{tabular}{|c|c|c|c|c|c|c|c|}
\hline 11 & $\begin{array}{l}\text { Bischofia javan- } \\
\text { ica Blume }\end{array}$ & $\begin{array}{l}\text { Euphorbi- } \\
\text { aceae }\end{array}$ & Root & $\begin{array}{l}\text { Stomach } \\
\text { infections, } \\
\text { UTIs }\end{array}$ & Decoction & Antibacterial & $\begin{array}{l}\text { Rajbongshi et al.(2014), } \\
\text { Guptaetal.(1988) }\end{array}$ \\
\hline 12 & Brassica nigra $\mathrm{L}$. & $\begin{array}{l}\text { Brassica- } \\
\text { ceae }\end{array}$ & Seed & UTIs & $\begin{array}{l}\text { Seeds are } \\
\text { grinded to take }\end{array}$ & $\begin{array}{l}\text { Flavonoids, alkaloids, } \\
\text { Sterols, Saponins, } \\
\text { Glycosides, Steroids, } \\
\text { Tannins, }\end{array}$ & Mirzaii M, et al., (2018) \\
\hline 13 & $\begin{array}{l}\text { Caesalpinia } \\
\text { decapetala } \\
\text { (Roth)Alston }\end{array}$ & $\begin{array}{l}\text { Caesal- } \\
\text { piniaceae }\end{array}$ & Root & $\begin{array}{l}\text { Skin in- } \\
\text { fections, } \\
\text { UTIs }\end{array}$ & Decoction & Antioxidant & $\begin{array}{l}\text { Weietal.(2013); Pawar \& } \\
\text { Surana(2010) }\end{array}$ \\
\hline 14 & $\begin{array}{l}\text { Caesalpinia nuga } \\
\text { (L.) }\end{array}$ & Fabaceae & $\begin{array}{l}\text { Leaves, } \\
\text { root }\end{array}$ & UTIs & $\begin{array}{l}\text { Powder of root } \\
\text { and leaves }\end{array}$ & $\begin{array}{l}\text { Flavonoids, Carbohy- } \\
\text { drates, Glycosides, } \\
\text { Phenols, Saponins, } \\
\text { Tannins }\end{array}$ & Wojnicz D, (2012) \\
\hline 15 & $\begin{array}{l}\text { Camellia sinensis } \\
\text { L. }\end{array}$ & Theaceae & Leaves & UTIs & $\begin{array}{l}\text { Dry unpro- } \\
\text { cessed leaves, } \\
\text { spray-dried } \\
\text { aqueous extract }\end{array}$ & $\begin{array}{l}\text { Phenolic compounds, } \\
\text { glycosides, alkaloids }\end{array}$ & Kasote DM, (2017) \\
\hline 16 & $\begin{array}{l}\text { Carissa edulis } \\
\text { Vahl }\end{array}$ & $\begin{array}{l}\text { Apocyna- } \\
\text { ceae }\end{array}$ & Root & UTIs & Decoction & $\begin{array}{l}\text { Anti-plasmodial } \\
\text { (P.falciparum) }\end{array}$ & Kebeneietal.(2011) \\
\hline 17 & $\begin{array}{l}\text { Cassia didy- } \\
\text { mobotrya Fresen. }\end{array}$ & Fabaceae & Leaves & $\begin{array}{l}\text { Stomach, } \\
\text { skin, oral } \\
\text { Cavity in- } \\
\text { fections, } \\
\text { UTIs }\end{array}$ & Poultice & Antimicrobial & $\begin{array}{lcl}\text { Boily } \quad \& & \text { Van- } \\
\text { Puyvelde(1986) } & \end{array}$ \\
\hline 18 & $\begin{array}{l}\text { Cichorium inty- } \\
\text { bus L. }\end{array}$ & $\begin{array}{l}\text { Aster- } \\
\text { aceae }\end{array}$ & Leaves & UTIs & $\begin{array}{l}\text { Powder of } \\
\text { leaves is }\end{array}$ & $\begin{array}{l}\text { taken Flavonoids, } \\
\text { Terpenoids, Tannins, } \\
\text { Saponins, Cardiac } \\
\text { glycosides }\end{array}$ & Rafsanjany N, (2013) \\
\hline 19 & $\begin{array}{l}\text { Clerodendrum } \\
\text { myricoides } \\
\text { (Hochst) R.Br. }\end{array}$ & $\begin{array}{l}\text { Lami- } \\
\text { aceae }\end{array}$ & Root & $\begin{array}{l}\text { Stomach } \\
\text { infections, } \\
\text { UTIs }\end{array}$ & Decoction & $\begin{array}{l}\text { Antibacterial, Anti- } \\
\text { fungal, Anti-plasmo- } \\
\text { dial }\end{array}$ & $\begin{array}{l}\text { Mulaudzi etal.(2012); } \\
\text { Muregietal.(2004) }\end{array}$ \\
\hline 20 & $\begin{array}{l}\text { Clitoria ternatea } \\
\text { L. }\end{array}$ & Fabaceae & Root & $\begin{array}{l}\text { UTIs, skin } \\
\text { infection }\end{array}$ & $\begin{array}{l}\text { Special prepa- } \\
\text { ration with rice } \\
\text { water is made to } \\
\text { take its roots }\end{array}$ & $\begin{array}{l}\text { Phenols, Flavonoids, } \\
\text { Saponins }\end{array}$ & Chang SS, et al., (1999) \\
\hline 21 & $\begin{array}{l}\text { Croton } \\
\text { macrostachyus } \\
\text { Hochst ex Delile }\end{array}$ & $\begin{array}{l}\text { Euphorbi- } \\
\text { aceae }\end{array}$ & Bark & $\begin{array}{l}\text { Oral cav- } \\
\text { ity infec- } \\
\text { tions, } \\
\text { UTIs }\end{array}$ & $\begin{array}{l}\text { Decoction for } \\
\text { gargle }\end{array}$ & Antimicrobial & Lulekal et al.(2013) \\
\hline 22 & $\begin{array}{l}\text { Cucumis sativus } \\
\text { L. }\end{array}$ & $\begin{array}{l}\text { Cucurbi- } \\
\text { taceae }\end{array}$ & Seed & UTIs & $\begin{array}{l}\text { Grinded seeds } \\
\text { with rock salt } \\
\text { are taken }\end{array}$ & $\begin{array}{l}\text { Cardiac glycosides, } \\
\text { Tannins, Phytosterol, } \\
\text { Terpenoids, Sapo- } \\
\text { nins, }\end{array}$ & Rosin MP. (1992) \\
\hline 23 & $\begin{array}{l}\text { Dichrocephala } \\
\text { integrifolia }\end{array}$ & $\begin{array}{l}\text { Aster- } \\
\text { aceae }\end{array}$ & Leaves & $\begin{array}{l}\text { Oral cav- } \\
\text { ity infec- } \\
\text { tions, } \\
\text { UTIs }\end{array}$ & $\begin{array}{l}\text { Poultice, decoc- } \\
\text { tion }\end{array}$ & $\begin{array}{l}\text { Antimicrobial, anti- } \\
\text { oxidant, inhibits } \alpha- \\
\text { glucosidase }\end{array}$ & $\begin{array}{l}\text { Zhu. (2012); Zhu } \\
\text { etal.(2010); Kuiatee- } \\
\text { tal.(1999) }\end{array}$ \\
\hline 24 & $\begin{array}{l}\text { Erythrina abys- } \\
\text { sinica Lam. }\end{array}$ & Fabaceae & Bark & UTIs & Decoction & Antiviral & Mohammed etal.(2012) \\
\hline
\end{tabular}




\begin{tabular}{|c|c|c|c|c|c|c|c|}
\hline 25 & $\begin{array}{l}\text { Hibiscus } \quad \text { rosa- } \\
\text { sinensis L. }\end{array}$ & $\begin{array}{l}\text { Malva- } \\
\text { ceae }\end{array}$ & Flower & UTIs & $\begin{array}{l}\text { Decoction of } \\
\text { flower is per- } \\
\text { formed before } \\
\text { taken }\end{array}$ & $\begin{array}{l}\text { Flavonoides, Ster- } \\
\text { oids, Tannins, Glyco- } \\
\text { sides, Phenols, Sapo- } \\
\text { nins, Phlobatannins, } \\
\text { Terpenoids, }\end{array}$ & Vicariotto F., (2014) \\
\hline 26 & $\begin{array}{l}\text { Leonotis nepeti- } \\
\text { folia }(\mathrm{L}) \mathrm{R} . \mathrm{Br} .\end{array}$ & $\begin{array}{l}\text { Lami- } \\
\text { aceae }\end{array}$ & Leaves & $\begin{array}{l}\text { Stomach } \\
\text { infections, } \\
\text { UTIs }\end{array}$ & Ash & $\begin{array}{l}\text { Antimicrobial, anti- } \\
\text { oxidative }\end{array}$ & Sobolewska et al.(2012) \\
\hline 27 & $\begin{array}{l}\text { Malva sylvestris } \\
\mathrm{L}\end{array}$ & $\begin{array}{l}\text { Malva- } \\
\text { ceae }\end{array}$ & Leaves & UTIs & Used in salad & $\begin{array}{l}\text { Alkaloids, Tannins, } \\
\text { Phenols, Flavonoides, } \\
\text { Saponins, }\end{array}$ & $\begin{array}{l}\text { Duraipandiyan \& Ignaci- } \\
\text { muthu(2009) }\end{array}$ \\
\hline 28 & $\begin{array}{l}\text { Oxalis cornicu- } \\
\text { lata } \mathrm{L} .\end{array}$ & $\begin{array}{l}\text { Oxalida- } \\
\text { ceae }\end{array}$ & Leaves & $\begin{array}{l}\text { Skin in- } \\
\text { fections, } \\
\text { UTIs }\end{array}$ & Poultice & $\begin{array}{l}\text { Antifungal, antioxi- } \\
\text { dative }\end{array}$ & $\begin{array}{l}\text { Lagnika etal.(2014); } \\
\text { Aruna et al.(2014) }\end{array}$ \\
\hline 29 & $\begin{array}{l}\text { Pimpinella ani- } \\
\text { sum L. }\end{array}$ & Apiaceae & Seed & UTIs & $\begin{array}{l}\text { Seeds as such } \\
\text { are taken }\end{array}$ & $\begin{array}{l}\text { Alkaloids, Flavo- } \\
\text { noids, Cardiac Glyco- } \\
\text { sides, Terpenoids, } \\
\text { Carbohydrate, Phy- } \\
\text { tosterols }\end{array}$ & Nadir M, (2013) \\
\hline 30 & Prunella Vulgaris & $\begin{array}{l}\text { Lami- } \\
\text { aceae }\end{array}$ & $\begin{array}{l}\text { Leaves, } \\
\text { Stem }\end{array}$ & UTIs & Salads & $\begin{array}{l}\text { Phytosteroids, tan- } \\
\text { nins, lupeol, D-cam- } \\
\text { phor, fenchone, cya- } \\
\text { nidin, delphinidin, } \\
\text { beta-sitosterol, }\end{array}$ & $\begin{array}{l}\text { Khosravi AD, et al., } \\
(2014)\end{array}$ \\
\hline 31 & $\begin{array}{l}\text { Rhoicissus tri- } \\
\text { dentata (L.f) } \\
\text { Wild \& Drum }\end{array}$ & Vitaceae & Leaves & $\begin{array}{l}\text { Stomach, } \\
\text { oral cavity } \\
\text { infections, } \\
\text { UTIs }\end{array}$ & $\begin{array}{l}\text { Decoction, Ash } \\
\text { decoction }\end{array}$ & $\begin{array}{l}\text { Antibacterial, Anti- } \\
\text { fungal, Anti-inflam- } \\
\text { matory }\end{array}$ & Lin etal. (1999) \\
\hline 32 & $\begin{array}{l}\text { Rhus natalensis } \\
\text { Bernh. Ex Krauss }\end{array}$ & $\begin{array}{l}\text { Anacardi- } \\
\text { aceae }\end{array}$ & Bark & UTIs & Decoction & $\begin{array}{l}\text { Antimicrobial, diar- } \\
\text { rhoeal infections }\end{array}$ & $\begin{array}{l}\text { Mwangietal. (2013); Ko- } \\
\text { riretal.(2012); Ka- } \\
\text { matenesi et al. -2014 }\end{array}$ \\
\hline 33 & $\begin{array}{l}\text { Rubia cordifolia } \\
\text { L. }\end{array}$ & $\begin{array}{l}\text { Rubia- } \\
\text { ceae }\end{array}$ & Leaves & $\begin{array}{l}\text { Skin in- } \\
\text { fection, } \\
\text { UTIs }\end{array}$ & Poultice & Antibacterial & $\begin{array}{l}\text { Ibraheimand Gouda } \\
(2010) \text {; Ibraeimand Ah- } \\
\text { med (2009); Ibraheim } \\
\text { (2002); Qiao et al.(1990) }\end{array}$ \\
\hline 34 & $\begin{array}{l}\text { Spilanthes mauri- } \\
\text { tiana DC. }\end{array}$ & $\begin{array}{l}\text { Aster- } \\
\text { aceae }\end{array}$ & Leaves & $\begin{array}{l}\text { Oral cav- } \\
\text { ity infec- } \\
\text { tions, } \\
\text { UTIs }\end{array}$ & $\begin{array}{l}\text { Decoction For } \\
\text { gargle }\end{array}$ & $\begin{array}{l}\text { Antibacterial and an- } \\
\text { tiviral }\end{array}$ & Cos et al. (2002) \\
\hline 35 & $\begin{array}{l}\text { Syzygium cumini } \\
\text { (L.) Skeels }\end{array}$ & $\begin{array}{l}\text { Myr- } \\
\text { taceae }\end{array}$ & Bark & UTIs & $\begin{array}{l}\text { Extract of Bark } \\
\text { is taken }\end{array}$ & $\begin{array}{l}\text { Flavonoids, Phy- } \\
\text { tosterols, Steroids, al- } \\
\text { kaloids, Amino acid, } \\
\text { Cardiac glycosides, } \\
\text { Saponins, Phenols, } \\
\text { Tannins, Terpenoids }\end{array}$ & $\begin{array}{l}\text { Duraipandiyan \& Ignaci- } \\
\text { muthu (2009) }\end{array}$ \\
\hline 36 & $\begin{array}{l}\text { Toddalia asiatica } \\
\text { (L.) Lam }\end{array}$ & Rutaceae & Root & UTIs & $\begin{array}{l}\text { Boiling, decoc- } \\
\text { tion }\end{array}$ & $\begin{array}{l}\text { Antibacterial, anti- } \\
\text { fungal }\end{array}$ & $\begin{array}{l}\text { Madhavan et al. (2012); } \\
\text { Karunai et al. (2012); } \\
\text { Orwa et al.(2008); Ishii et } \\
\text { al.(1991) }\end{array}$ \\
\hline 37 & Urtica dioica $\mathrm{L}$. & $\begin{array}{l}\text { Urtica- } \\
\text { ceae }\end{array}$ & Leaves & $\begin{array}{l}\text { Skin and } \\
\text { stomach } \\
\text { infections, } \\
\text { UTIs }\end{array}$ & Poultice, ashash & $\begin{array}{l}\text { Antibacterial, anti- } \\
\text { fungal, diuretic }\end{array}$ & Bahmani M, et al., (2016) \\
\hline
\end{tabular}




\begin{tabular}{|l|l|l|l|l|l|l|l|}
\hline 38 & Vigna mungo L. & Fabaceae & Seed & $\begin{array}{l}\text { UTIs, skin } \\
\text { infection }\end{array}$ & Seeds are taken & $\begin{array}{l}\text { Flavonoids, Alka- } \\
\text { loids, Phenols, Ascor- } \\
\text { bic acid, Steroids, } \\
\text { Tannins Glycosides, } \\
\text { Saponins }\end{array}$ & $\begin{array}{l}\text { Duthu (2009) } \\
\text { muandiyan \& Ignaci- }\end{array}$ \\
\hline 39 & $\begin{array}{l}\text { Zizyphus jujuba } \\
\text { Mill. }\end{array}$ & $\begin{array}{l}\text { Rham- } \\
\text { naceae }\end{array}$ & Fruit & $\begin{array}{l}\text { UTIs, skin } \\
\text { infection }\end{array}$ & $\begin{array}{l}\text { Extract of the } \\
\text { fruit is taken }\end{array}$ & $\begin{array}{l}\text { Alkaloids, Glyco- } \\
\text { sides, Flavonoids, } \\
\text { Saponins, Phenolic, } \\
\text { Terpenoids }\end{array}$ & Bahmani M, et al., (2016) \\
\hline
\end{tabular}

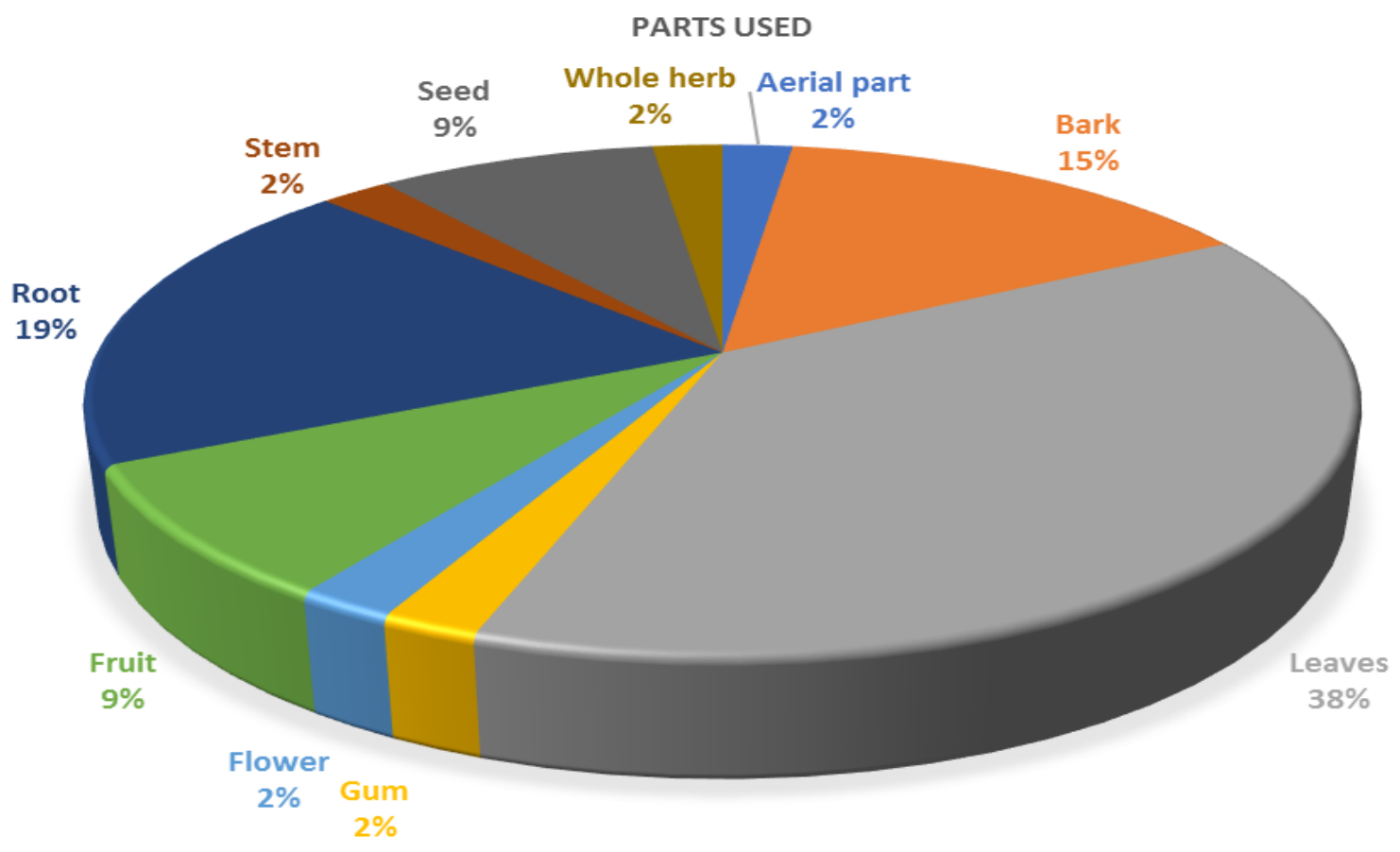

Chart 1: Part used of Medicinal plants which are used in UTI 
Chart 2: List of Families used in UTI

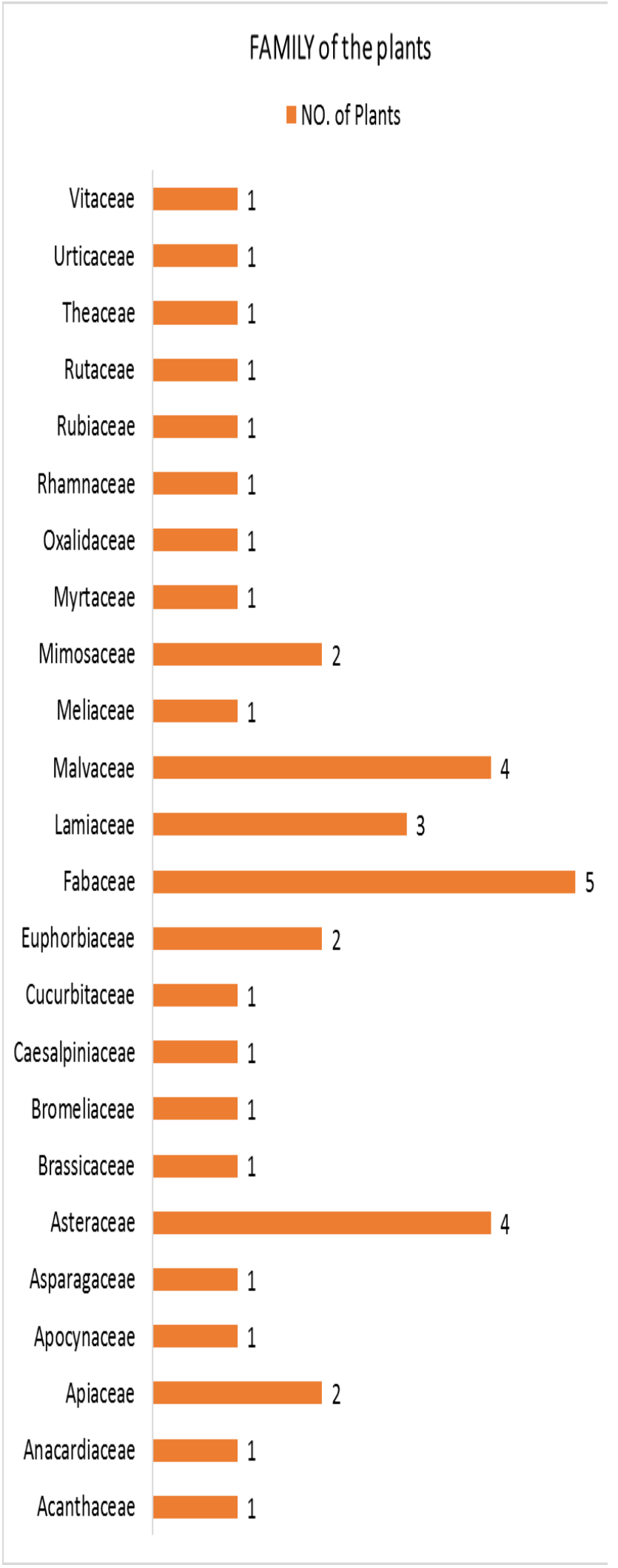

In this study showed, the Fabaceae family's plants were 5, Asteracea and Malvaceae family plants were 4, Lamiaceae family's plants were 3, Apiaceae, Euphorbiaceae and Mimosaceae family's plants were 2. Other 1 plant in various 17 families out of the 24 families.

\section{DISCUSSION}

Neerchurukku is classified under Neerinai Arukkal noigal therefore, we have to treat the Neerchurukku patient with siddha medicines especially herbal preparation which were single materials or compound medicines. It is notified in siddha literatures already by their clinical trials. Herbal remedy for Neerchurukku is essential one with cost effective and less harmful naturally available plants source management. Therefore, this research is going to explore the siddha knowledge and reveal the siddha system to the world.

In this research concluded as; Fabaceae family's plants were 5, Asteracea and Malvaceae family plants were 4, Lamiaceae family's plants were 3, Apiaceae, Euphorbiaceae and Mimosaceae family's plants were 2. Other 1 plant in various 17 families out of the 24 families. The plant parts used in the management of UTS as; leaves were most used in 18 plants $(38 \%)$, roots were used in 9 plants (19\%), barks were 7 plants (15\%), 4 plants of fruits and seeds used (9\%) and aerial parts, whole plants, stem flower and gum were used by only one plant $(2 \%)$.

The all medicines were proved, and authenticated actions are Antibacterial, Antifungal, Anti-plasmodial, Antimicrobial, Antioxidant, inhibits $\alpha$-glucosidase, Antiviral and Anti-inflammatory. All medicinal plants having Phenols, Flavonoids, Saponins, Cardiac glycosides, Tannins, Phytosterol, Terpenoids, Steroids, Glycosides, Anthraquinones, Terpenes, Carbohydrate, Fenchone, Cyanidin, Delphinidin, Beta-Sitosterol, Phytosterols, Alkaloids, Amino acid and Phloba-tannins of the selected plants.

Form of administration of plant such as; With sugar seedless fruits taken orally, Decoction, Gum Paste and leaves with cow's milk are taken orally, Powder of Bark, Extract of fresh leaves and fruits, Powder of bark and leaves, fresh fruits are taken, Extract of entire plants is taken, Seeds are grinded to take, Powder of 
root and leaves, Dry unprocessed leaves, spray-dried aqueous extract, Special preparation with rice water is made to take its roots, Decoction for gargle, Used in salad, Poultice and Ash decoction.

\section{CONCLUSION}

This research finally concluded as; collected and reviewed the 39 medicinal plants used in the management of Neerchurukku (UTI). listed out the 39 medicinalplants used for management of Neerchurukku. Fabaceae family's plants were 5, Asteracea and Malvaceae family plants were 4, Lamiaceae family's plants were 3, Apiaceae, Euphorbiaceae and Mimosaceae family's plants were 2. Other 1 plant in various 17 families out of the 24 families. Plant's Parts used for management of UTS as; leaves were most used in 18 plants (38\%), roots were used in 9 plants (19\%), barks were 7 plants $(15 \%), 4$ plants of fruits and seeds used (9\%) and aerial parts, whole plants, stem flower and gum were used by only one plant $(2 \%)$.

Documented Antibacterial, Antifungal, Anti-plasmodial, Antimicrobial, Antioxidant, inhibits $\alpha$-glucosidase, Antiviral and Anti-inflammatory pharmacological actions of plants which are used for management of Neerchurukku.

\section{Acknowledgement}

I express my sincere thanks to Lecturers and friends of Department of General medicine, Government Siddha medical Collage \& Hospital, Palayamkottai

\section{REFERENCES}

1. Liu P. [Care of patients with acute urinary tract infection treated by Chinese herbal drugs]. Zhonghua $\mathrm{Hu}$ Li Za Zhi. 1987 Mar;22(3):116-7. Chinese. PMID: 3650136.

2. WISSMANNS HG. [On the treatment of nonspecific diseases of the urinary tract with a new type of phytotherapeutic]. Ther Ggw. 1962 Jan; 101:26-8. German. PMID: 14007705.

3. KRUSEN F. [On phytotherapy of inflammatory diseases of the urinary tract]. Hippokrates. 1961 Oct 31; 32:819-20. German. PMID: 14459959.

4. Rokia S, Drissa D, Seydou D, Colette E, Flabou B. Activite antibacterienne et antalgique de deux recettes traditionnelles utilisees dans le traitement des infections urinaires et la cystite au Mali [Antibacterial and anti-algal activity if two traditional drugs in the treatment of urinary tract infection and cystitis in Mali]. Mali Med. 2006;21(1):18-24. French. PMID: 17390524.

5. Peng Y, Yan H, Wang SQ, Liu XT. 65 cases of urinary tract infection treated by total acid of Achillea alpina. J Tradit Chin Med. 1983 Sep;3(3):217-8. PMID: 6355666.

6. Dou GX. [196 cases of urinary tract infection treated with traditional Chinese medicine and Western medicine combined]. Zhong Xi Yi Jie He Za Zhi. 1985 Sep;5(9):526-9, 514. Chinese. PMID: 2936510.

7. Vogel A. Die Behandlung von Harnwegsinfektionen mit pflanzlichen Wirkstoffen [Management of urinary tract infections using plant extracts]. ZFA (Stuttgart). 1979 Feb 20;55(5):343-6. German. PMID: 425648.

8. Beuthe D. Die phytotherapie der harnweginfektionen in der gynaekologie [phytotherapy of urinary tract infections in gynecology]. Arztl Forsch. 1964 Oct 10; 18:547-9. German. PMID: 14325252.

9. Narayanan AS, Raja SS, Ponmurugan K, Kandekar SC, Natarajaseenivasan K, Maripandi A, Mandeel QA. Antibacterial activity of selected medicinal plants against multiple antibiotic resistant uropathogens: a study from Kolli Hills, Tamil Nadu, India. Benef Microbes. 2011 Sep;2(3):235-43. doi: 10.3920/BM2010.0033. PMID: 21986363.

10. Buchli A, Trüb B. Phytotherapie bei Harnwegsinfekten. Heilpflanzenanwendungen: einfach und wirksam [Phytotherapy in urinary tract infections. Using medicinal plants: simple and effective]. Krankenpfl Soins Infirm. 2011;104(3):26-7. German. PMID: 21417053.

11. Gross AJ, Hummel G. Goethe almost died of urosepsis. World J Urol. 1999 Dec;17(6):421-4. doi: 10.1007/s003450050169. PMID: 10654374.

12. Eisele h. Die bedeutung unspezifischer mittel bei der behandlung von harnwegsinfektionen in klinik und praxis [significance of non-specific drugs in the treatment of urinary tract infections in clinical and private practice]. Med Welt. 1965 Feb 6; 14:315-7. German. PMID: 14264349.

13. Vogel NW, Taschetto AP, Dall'agnol R, Weidlich L, Ethur EM. Assessment of the antimicrobial effect of 
three plants used for therapy of community-acquired urinary tract infection in Rio Grande do Sul (Brazil). J Ethnopharmacol. 2011 Oct 11;137(3):1334-6. doi: 10.1016/j.jep.2011.07.070. Epub 2011 Aug 5. PMID:21843617.

14. Pereira RS, Sumita TC, Furlan MR, Jorge AO, Ueno M. Atividade antibacteriana de óleos essenciais em cepas isoladas de infecção urinária [Antibacterial activity of essential oils on microorganisms isolated from urinary tract infection]. Rev Saude Publica. 2004 Apr;38(2):326-8. Portuguese. doi: 10.1590/s0034-89102004000200025. Epub 2004 Apr 26. PMID: 15122392.

15. Ritchie BF. Empowering female residents: alternative approaches to urinary tract infections (UTIs) in nursing homes. Aust J Holist Nurs. 2000 Apr;7(1):26-30. PMID: 11898217.

16. Steger W, Haase W. Zur Wirksamkeit und Verträglichkeit von Blasen-Nieren-Tee Uroflux bei der Behandlung von Harnwegsinfektionen speziell bei Diabetes mellitus [Efficacy and tolerance of Uroflux, a bladder and kidney tea, in the treatment of urinary tract infections, especially in diabetes mellitus]. Fortschr Med. 1979 Nov 15;97(43):2007-10. German. PMID: 574483.

17. Pagonas N, Hörstrup J, Schmidt D, Benz P, Schindler R, Reinke P, van der Giet M, Zidek W, Westhoff TH. Prophylaxis of recurrent urinary tract infection after renal transplantation by cranberry juice and L-methionine. Transplant Proc. 2012 Dec;44(10):3017-21. doi: 10.1016/j.transproceed.2012.06.071. PMID: 23195017.

18. Menchini-Fabris GF, Giorgi P, Andreini F, Canale D, Paoli R, Sarteschi ML. Nuove prospettive di impiego del Pygeum Africanum nella patologia prostato- vescicolare [New perspectives on the use of Pygeum Africanum in prostato-bladder pathology]. Arch Ital Urol Nefrol Androl. 1988 Sep;60(3):313-22. Italian. PMID: 2975866.

19. Van Cappellen. Le traitement de la tuberculose urogénitale par chaulmoogra et streptomycine. Brux Med. 1948 Aug 1;28(31):1604. Undetermined Language. PMID: 18934331.

20. Beydokthi SS, Sendker J, Brandt S, Hensel A. Traditionally used medicinal plants against uncomplicated urinary tract infections: Hexadecyl coumaric acid ester from the rhizomes of Agropyron repens (L.) P. Beauv. with antiadhesive activity against uropathogenic E. coli. Fitoterapia. 2017 Mar; 117:22-27. doi: 10.1016/j.fitote.2016.12.010. Epub 2016 Dec 29. PMID: 28040531.

21. Datla R, Rao SR, Murthy KJ. Excretion studies of nitrofurantoin and nitrofurantoin with deglycyrrhizinated liquorice. Indian J Physiol Pharmacol. 1981 Jan-Mar;25(1):59-63. PMID: 7024125.

22. Okragla E, Chraniuk M, Wolska L. Microtox Test as a tool to Assess Antimicrobial Properties of Herbal Infusions Used In Urinary Tract Infections. Acta Pol Pharm. 2017 May;74(3):895-901. PMID: 29513959.

23. Omwenga EO, Hensel A, Shitandi A, Goycoolea FM. Ethnobotanical survey of traditionally used medicinal plants for infections of skin, gastrointestinal tract, urinary tract and the oral cavity in Borabu sub-county, Nyamira county, Kenya. J Ethnopharmacol. 2015 Dec 24; 176:508-14. doi: 10.1016/j.jep.2015.11.032. Epub 2015 Nov 17. PMID: 26596256.

24. Schnell UC, Thelen MJ. Untersuchungen über die antibakterielle Wirkung von Carito in der gynäkologischen Urologie [Antibacterial effect of Carito in gynecologic urology]. MMW Munch Med Wochenschr. 1977 Jan 28;119(4):127-8. German. PMID: 402557.

25. Gallien P, Amarenco G, Benoit N, Bonniaud V, Donzé C, Kerdraon J, de Seze M, Denys P, Renault A, Naudet F, Reymann JM. Cranberry versus placebo in the prevention of urinary infections in multiple sclerosis: a multicenter, randomized, placebo-controlled, double-blind trial. Mult Scler. 2014 Aug;20(9):1252-9. doi: 10.1177/1352458513517592. Epub 2014 Jan 8. PMID: 24402038.

26. Rafsanjany N, Sendker J, Lechtenberg M, Petereit F, Scharf B, Hensel A. Traditionally used medicinal plants against uncomplicated urinary tract infections: Are unusual, flavan-4-ol- and derhamnosylmaysin derivatives responsible for the antiadhesive activity of extracts obtained from stigmata of Zea mays L. against uropathogenic E. coli and Benzethonium chloride as frequent contaminant faking potential antibacterial activities? Fitoterapia. 2015 Sep; 105:24653. doi: 10.1016/j.fitote.2015.07.014. Epub $2015 \mathrm{Jul}$ 22. PMID: 26210697. 
27. Zhang LF, Zhao JX. [The recent research situation of Euonymus alatus]. Zhongguo Zhong Yao Za Zhi. 2005 Dec;30(24):1895-8. Chinese. PMID: 16494017.

28. Anparasu. K., Yugi Vaidya Chinthamani- 800, 1st edition, 1998.

\section{Source of Support: Nil \\ Conflict of Interest: None Declared}

How to cite this URL: Kavery Piratheepkumar et al: A Systematic Review On Selected Medicinal Plants Used In The Management Of Neerchurukku (UTI). International Ayurvedic Medical Journal \{online\} 2021 \{cited March, 2021\} Available from:

http://www.iamj.in/posts/images/upload/519 528.pdf 